\title{
Regularity Theories Reassessed
}

\author{
Michael Baumgartner
}

Received: 3 April 2007 / Revised: 24 November 2007 / Accepted: 3 December 2007 /

Published online: 9 January 2008

(C) Springer Science + Business Media B.V. 2007

\begin{abstract}
For a long time, regularity accounts of causation have virtually vanished from the scene. Problems encountered within other theoretical frameworks have recently induced authors working on causation, laws of nature, or methodologies of causal reasoning - as e.g. May (Kausales Schliessen. Eine Untersuchung über kausale Erklärungen und Theorienbildung. Ph.D. thesis, Universität Hamburg, Hamburg, 1999), Ragin (Fuzzy-set social science. Chicago: University of Chicago Press, 2000), Graßhoff and May (Causal regularities. In W. Spohn, M. Ledwig, \& M. Esfeld (Eds.), Current issues in causation (pp. 85-114). Paderborn: Mentis, 2001), Swartz (The concept of physical law (2nd ed.). http://www.sfu.ca/philosophy/physical-law/, 2003), Halpin (Erkenntnis, 58, 137-168, 2003) - to direct their attention back to regularity theoretic analyses. In light of the latest proposals of regularity theories, the paper at hand therefore reassesses the criticism raised against regularity accounts since the INUS theory of causation of Mackie (The cement of the universe. A study of causation. Oxford: Clarendon Press, 1974). It is shown that most of these objections target strikingly over-simplified regularity theoretic sketches. By outlining ways to refute these objections it is argued that the prevalent conviction as to the overall failure of regularity theories has been hasty.
\end{abstract}

Keywords Causation - Regularity theory • Determinism • David Hume • John L. Mackie

\footnotetext{
M. Baumgartner $(\bowtie)$

Department of Philosophy, University of Bern,

Länggassstr. 49a, 3012 Bern, Switzerland

e-mail: baumgartner@philo.unibe.ch
} 


\section{Introduction}

A mere glance at the abundance of controversial literature on causation published during the past 30 years reveals that regularity accounts of causation - until recently - virtually vanished from the scene. For lack of space and interest, studies not primarily concerned with causation every now and then roughly explicated our causal intuitions in terms of regularities, but hardly anybody seriously wanting to analyze causation resorted to regularity accounts any more. Problems encountered within other theoretical frameworks have recently induced philosophers working on causation, laws of nature, or methodologies of causal reasoning - as e.g. May (1999), Ragin (2000), Graßhoff and May (2001), Swartz (2003), or Halpin (2003) - to direct their attention back to regularity theoretic analyses. In light of the latest proposals of regularity theories as can be found in Graßhoff and May (2001) and Baumgartner and Graßhoff (2004), the paper at hand therefore reassesses the criticism raised against regularity accounts since Mackie's famous, yet failed, (1974) attempts at analyzing causation with recourse to regularities among types of events.

Notwithstanding the skepticism encountered by Hume's regularity theoretic successors, there are several commonly acknowledged advantages of an analysis of causation in terms of regularities. A regularity theoretic notion of causation directly mirrors central pre-theoretic intuitions with respect to the cause-effect relation as e.g. expressed in "The same cause is always accompanied by the same effect". The conceptual apparatus resorted to by a regularity theoretic analysis is fully embedded within the uncontroversial and well mastered area of extensional standard logic. ${ }^{1}$ Furthermore, unlike e.g. counterfactual accounts regularity theories straightforwardly handle cases of overdetermination. As against interventionist or manipulatory accounts, analyses of causation in terms of regularities do not run the risk of being anthropocentric. Contrary to probabilistic accounts, regularity theories are not compromised by paradoxical data as, for instance, generated in cases of Simpson's Paradox. Finally, while transference theories treat a fundamental type of causal process, transference processes, as conceptually primitive and thus do not attempt to provide a reductive analysis of causation, regularity accounts, properly conceived, offer the promising prospect of explicating causation in entirely non-causal terms.

Hence, the pages to come shall review the objections that have traditionally been raised against regularity accounts. It will be shown that most of these objections target strikingly over-simplified regularity theoretic sketches. By outlining ways to refute these objections it will be argued that the prevalent conviction as to the overall failure of regularity theories has been hasty - to say the least.

\footnotetext{
${ }^{1}$ There are some analyses of causation referred to as "regularity theories" that are stated in terms of nomic sufficiency which cannot be spelled out by means of first-order logic, but presupposes some modal system (cf. e.g. Hausman 1998, pp. 42-43). This terminology, however, blurs the important distinction between empiricist and modal analyses. Such as not to drop this distinction the label "regularity theory" is reserved for first-order analyses in this paper.
} 


\section{Hume's Legacy}

The philosophical core of regularity theories of causation consists of three main tenets: (1) the causal relation is not an ontological primitive, (2) general causation - causation on type level - is the primary analysandum, ${ }^{2}$ and (3) universal regularities among event types or factors constitute the primary analysans. There are - at least - two causal relations, one on type and another on token level. "Drinking is a cause of drunkenness" is a case of general causation, i.e. causation among factors, while "Shamus' drinking of 6 beers at noon on September 7, 2004 causes Shamus' drunkenness in the afternoon of September 7, 2004" relates token events and, accordingly, is a case of singular causation. A factor that is related to another factor in terms of general causation is said to be causally relevant to the latter. For brevity, if a first factor is causally relevant to a second factor, the first can also be referred to as a cause of the second. Moreover, such as to avoid unnecessary terminological complications, I shall often simply speak of causation whenever both causal relations are at issue or whenever the context clarifies whether singular or general causation is under consideration. Factors are taken to be similarity sets of event tokens. They are sets of type identical token events, of events that share at least one feature. Contrary to token events, event types are generic entities. Whenever a member of a similarity set that corresponds to an event type occurs, the latter is said to be instantiated.

According to Hume, the godfather of regularity theories, single event sequences are not identifiable as being of causal nature by some inherent physical feature or property. A causal interpretation of an event sequence is warranted only if the corresponding events, understood as spatiotemporally located tokens or particulars, ${ }^{3}$ instantiate factors one of which is related in terms of general causation to the other. Thus, as singular causation can be straightforwardly accounted for given an analysis of general causation, spelling out the latter relation constitutes the core goal of a regularity theory - hence tenet (2). For completeness, singular causation is separately accounted for in "Singular Causation" below.

Events are symbolized by italicized minuscules $a, b$, etc., with variables $x, y$, $x_{1}, x_{2}$, etc. running over the domain of events. Factors, on the other hand, are symbolized by italicized capital letters $A, B$, etc., with variables $Z, Z_{1}, Z_{2}$ etc. running over the domain of factors. An event type as "striking a match" can be

\footnotetext{
${ }^{2}$ There are some regularity theoretic proposals that do not subscribe to this tenet (e.g. Mackie 1965), but rather take singular causation to be primary. Some criticism raised against regularity theories over the past four decades targets this kind of singularist account (cf. Collins et al. (2004); Kim 1973; Davidson 1967). As the paper at hand is only concerned with regularity theories whose primary analysandum is general causation, these singularist objections will be disregarded in the present context (cf. also "Singular Causation" below).

${ }^{3}$ This focus on events does not straightforwardly cover cases of causally related absences or omissions. The problems posed by causal dependencies as between omitted vaccination and contracting influenza will be neglected in the present context. They are treated in Baumgartner (2006), Chap. 3. For interesting proposals on how to deal with causation among absences cf. also Collins et al. (2004).
} 
defined as the set consisting of all token events in the extension of the predicate "... is a striking of a match", i.e. as $\{x: x$ is a striking of a match $\}$. Factors are negatable. The negation of a factor $A$ is written thus: $\bar{A}$. $\bar{A}$ is simply defined as the complementary set of $A .{ }^{4}$ Alternatively, factors can be seen as binary variables that take the value 1 whenever an event of the corresponding type occurs and the value 0 whenever no such event occurs.

Generic causal dependencies are not one-to-one, but many-to-one dependencies. Or put differently, while effects correspond to single factors, causes are parts of whole causing complexes - complex causes. A complex cause only becomes causally effective if all of its constituents are co-instantiated, i.e. instantiated close-by or coincidently. Coincidently instantiated factors are termed coincidences. Coincidences can be seen as conjunctions of coincidently instantiated factors $A_{1} \wedge A_{2} \wedge \ldots \wedge A_{n}$, which for simplicity shall be abbreviated by a mere concatenation of the respective factors: $A_{1} A_{2} \ldots A_{n}{ }^{5}$ Ordinarily, only a small subset of members of a complex cause are known or of interest to a causal investigation. Variables $X_{1}, X_{2}$, etc. are used to run over unknown or neglected elements of complex causes, e.g. $A X_{1}, B X_{2}$. Regularity theories subscribe to the principle of determinism which stipulates that, if the same causes are instantiated, the same effects occur. Expressed in the regularity theoretic terminology that yields: Causes are sufficient for their effects, i.e. if $A X_{1}$ is a complex cause of $B, A X_{1} \rightarrow B$. Moreover, instances of factors do not cause themselves - no self-causation on token level-, and effects and causes are spatiotemporally proximate - no action at a distance. ${ }^{6}$ All of this yields a first Hume-inspired proposal for a regularity theoretic account of causal relevance:

(I) $\quad A$ is causally relevant to $B$ iff $A$ is part of a sufficient condition $A X_{1}$ of $B$, such that the instances of $A X_{1}$ and $B$ differ and are spatiotemporally proximate.

Note that the relational constraints (I) imposes on the instances of factors that are related in terms of general causation forestall a formalization of causal statements by means of simple propositional conditionals. These relational constraints can only be adequately represented by means of firstorder expressions, which are properly introduced in the Appendix. While the decisive theoretical work as regards the analysis of causal relevance is done by regularities existing among the factors involved in a causal structure, those

\footnotetext{
${ }^{4}$ Instead of factors or event types one may also speak of event properties, as long as the latter are spelled out in purely extensional terms. Since the notion of a property tends to give rise to farreaching questions as to the extensional definability of properties or to the existence of negative properties (cf. Zangwill 2003), I prefer the philosophically less biased notion of a factor.

${ }^{5}$ Such a mere propositional formalization of coincidences can be read in terms of "Factor $A_{1}$ is instantiated coincidently with $A_{2}$ and ... and $A_{n} "$.

${ }^{6}$ Hume originally required temporal succession, not mere proximity (cf. Hume 1748, p. 146). In accordance with the usual practice, causes and effects are here only required to be spatiotemporally proximate such as not to preclude the possibility of simultaneous or backward causation on a priori grounds (cf. "Non-Symmetry").
} 
relational constraints merely assure that a suitable subset of all regularities among corresponding factors is chosen as a starting point for causal analyses. The relational constraints delineate the causally interesting regularities from causally meaningless regularities as "Whenever there is a table, there is a table", "For every first human step on the lunar surface there is a first human non-stop balloon flight around the world", or "Whenever there is a soccer game, there is a sport event". Throughout the main part of this paper propositional conditionals are used as convenient abbreviations of the causally interesting regularities identified by the first-order expressions presented in the Appendix.

\section{Monotony}

Implementing regularities along the lines of (I) to identify generic causal dependencies does not amount to a feasible analysis of causal relevance, because there are regularities of the required type that are not amenable to a causal interpretation. One such type of regularities is due to the law of monotony: Antecedents of conditionals can be salva veritate supplemented by further conjuncts. Striking a match with a certain speed and thrust, factor $A$, dryness of its flammable head $(B)$, and presence of oxygen $(C)$ shall be assumed to be jointly sufficient for the corresponding match to catch fire $(D)$, i.e. $A B C \rightarrow D$. Yet, if $A, B$, and $C$ are jointly sufficient for the match to light, the combination of $A B C$ and singing a song is thus sufficient, too. Or formally:

$$
A B C \rightarrow D \vdash A B C X \rightarrow D,
$$

where $X$ stands for an arbitrary factor or conjunction of factors. This demonstrates that being a part of a sufficient condition, i.e. being a conjunct within a sufficient conjunction of factors, is by no means sufficient for being a cause of the corresponding conditioned factor.

Broad (1930) has been the first to propose a solution to this problem. $\mathrm{He}$ does not analyze causes to be mere parts of sufficient conditions, but rather to be non-redundant parts of such conditions. A non-redundant part of a sufficient condition can be spelled out - in purely logical terms ${ }^{7}$ - as a conjunct of a sufficient condition such that, if it is eliminated from that condition, the latter loses its sufficiency for a corresponding effect. Accordingly, complex causes must be taken to be minimally sufficient conjunctions of factors - a minimally sufficient conjunction being a conjunction that does not have sufficient proper parts.

\footnotetext{
${ }^{7}$ Not all critics of regularity accounts have taken note of these purely logical ways to minimalize sufficient conditions. For instance, in 1970 Brand and Swain still erroneously claimed that minimalizing sufficient conditions cannot be accomplished in non-causal and, thus, non-circular terms (cf. Brand and Swain 1970, p. 226).
} 
(II) $A$ is causally relevant to $B$ iff $A$ is part of a minimally sufficient condition $A X_{1}$ of $B$, such that the instances of $A X_{1}$ and $B$ differ and are spatiotemporally proximate.

Applying (II) to the match example prohibits a causal interpretation of the combination of striking a match, presence of oxygen, dryness of the match, and singing a song. The conjunction of these factors is merely sufficient, but not minimally sufficient for the match to catch fire. Requiring a minimalization of sufficient conditions in Broad's sense precludes a causal interpretation of arbitrary extensions of sufficient conditions based on the law of monotony.

\section{Empty Regularities}

Consider again the match example. The presence of oxygen, factor $C$, in combination with a properly struck match $(A)$ with a dry head $(B)$ is not the only minimally sufficient condition of the match catching fire $(D)$ that contains $C$. Another such condition is constituted by the presence of oxygen and the absence of oxygen: $C \bar{C}$. A contradiction is sufficient for any factor, not only for matches catching fire, but also for rain to fall and elephants to be born. That $C \bar{C}$ is moreover minimally sufficient for a match to light can easily be verified by either removing $C$ or $\bar{C}$, both of which is accompanied by a loss of sufficiency for $D$. More generally put: Material conditionals are true if their antecedents are false or non-instantiated, or empty for short. Any regularity statement $A X_{1} \rightarrow B$ whose antecedent is empty is, accordingly, termed an empty regularity. Empty regularities do not only result from logically non-instantiatable antecedents, but also from physically non-instantiatable antecedents as, for instance, "Whenever Pegasus goes skiing, Lake Thun is made of gold".

The truth of empty regularity statements raises another often cited problem for regularity accounts: Empty regularities are, notwithstanding their truth, not amenable to a causal interpretation. ${ }^{8}$ The combination of absence and presence of oxygen $-C \bar{C}$ - does not cause the sinking of Mississippi steamers, even though $\bar{C} \bar{C}$ in fact is minimally sufficient for these sinkings. Neither can Pegasus' ski tour be seen as a cause of the golden content of a lake.

Solutions to this problem are easily thought of. It is not the case that only a certain proper subset of all empty regularities consists of regularities that are not causally interpretable, rather, no empty regularities are thus interpretable. Causal dependencies only exist among causes and effects that are instantiated in nature. "Instantiated" in this context is not to be read in terms of "has occurred prior to a specific moment of investigation", but in terms of "has not occurred in all past and will not occur in all future". 9 Factors and conjunctions of factors may not be causally related if they have not been instantiated in all

\footnotetext{
${ }^{8}$ Cf. e.g. Armstrong (1983).

${ }^{9}$ Cf. the tenseless use of "exist" e.g. in Russell (1986), p. 217.

Springer
} 
past and will not be instantiated in all future. Therefore, empty regularities can straightforwardly be excluded from causal interpretability by adding a further constraint to (II) that requires the antecedent of causally interpretable regularities to be non-empty. ${ }^{10}$

(III) $A$ is causally relevant to $B$ iff the following conditions hold:

(1) $A$ is part of a minimally sufficient condition $A X_{1}$ of $B$,

(2) The instances of $A X_{1}$ and $B$ differ and are spatiotemporally proximate,

(3) There is an instance of $A X_{1}$.

\section{Non-Symmetry}

Another objection often raised against regularity accounts concerns the direction causation. The cause-effect relation is not symmetric and this lacking symmetry is usually claimed not to be adequately representable in regularity theoretic terms. In order to clarify what this criticism amounts to, some conceptual preliminaries are required. First, non-symmetry must be distinguished from asymmetry. A relation $C$ is non-symmetric iff Eq. 2 holds and asymmetric iff Eq. 3 holds. ${ }^{11}$

$$
\begin{aligned}
\neg \forall x \forall y(C x y \rightarrow C y x) & \text { or equivalently } \quad \exists x \exists y(C x y \wedge \neg C y x) \\
& \forall x \forall y(C x y \rightarrow \neg C y x)
\end{aligned}
$$

Every asymmetric relation with a non-empty extension is also non-symmetric, but not vice versa. Predicating asymmetry of a non-empty relation is a much stronger claim than predicating non-symmetry of it. It is undisputed that both general and singular causation are non-symmetric relations. There exist both general and singular causal dependencies and neither " $A$ is causally relevant to $B$ " implies " $B$ is causally relevant to $A$ " nor " $a$ causes $b$ " implies " $b$ causes $a$ ". Moreover, general causation clearly is not asymmetric, for there are event types that are causally relevant to themselves, as e.g. in causal cycles. For instance, with increasing unemployment the consumption of the population is reduced. This causes decreased profits on the side of the employers, which, in turn, causes them to lay off even more people. Thus, the unemployment increases anew. In contrast, on token level there are no events that cause themselves. Contrary to general causation, singular causation is irreflexive. Yet, whether it is moreover asymmetric is a question that is controversially

\footnotetext{
${ }^{10}$ Note that regularity theories of laws of nature are not as easily rendered immune to the empty regularities problem as regularity theories of causation. While factors without instances may not be claimed to be causally dependent, there may well be natural laws involving predicates with empty extensions, as e.g. “... travels faster than light” (cf. Molnar 1969).

${ }^{11}$ This terminology corresponds to logical and mathematical conventions (cf. e.g. Lemmon 1965, pp. 180-182). In addition to non-symmetry and asymmetry a relation may lack symmetry in terms of antisymmetry. A relation $C$ is antisymmetric iff $\forall x \forall y(x \neq y \wedge C x y \rightarrow \neg C y x)$.
} 
disputed in the literature. If event $a$ is a cause of event $b$, does it follow that $b$ is not a cause of $a$ ? Is there reciprocal causation on token level? ${ }^{12}$ For the purposes at hand this question can be postponed until "Singular Causation," for a regularity theory that focuses on general causation primarily needs to account for the latter's non-symmetry. In consequence, the criticism against regularity theories to the effect that they are not capable of adequately capturing the direction of causation amounts to the claim that the non-symmetry of general causation cannot be mirrored on regularity theoretic grounds. And indeed, prima facie there appears to be a problem.

$A$ being causally relevant to $B$ neither implies $B$ being causally relevant to $A$ nor $\bar{B}$ being causally relevant to $\bar{A}$. However, by contraposition $A \rightarrow B$ is a true regularity statement iff $\bar{B} \rightarrow \bar{A}$ is so too. Which of these conditionals is to be causally interpreted? It is certainly not the case that a factor is causally relevant to another factor iff the negation of the latter is causally relevant to the negation of the former. Accordingly, many critics of regularity theories have claimed that regularity accounts cannot adequately distinguish between causes and effects. ${ }^{13}$ (III) identifies $A$ to be a cause of $B$ iff it identifies $\bar{B}$ to be cause of $\bar{A}$, which indicates that we have not come up with an adequate analysis of causal relevance yet.

Satisfactorily mirroring the non-symmetry of general causation is an intricate problem any theory of causation has to solve. Normally the direction of causal relevance is accounted for with recourse to some non-symmetry that is external to the conceptual framework used in the analysans of causal relevance as - most prominently - the direction of time or human manipulation and intervention. ${ }^{14}$ Applied to the regularity theory considered here, this could possibly mean that conditionals in the sense of (III) are causally interpretable only if the instances of the antecedent precede the instances of the consequent. Along these lines, one of $A \rightarrow B$ and $\bar{B} \rightarrow \bar{A}$ could be excluded from causal interpretability. However, spelling out the non-symmetry of general causation by means of the direction of time presupposes that the latter is accounted for independently of the direction of causation, which runs counter to an often adopted program in the philosophy of time that takes the direction of causation to be primary. ${ }^{15}$ Furthermore, a temporal account of the direction of causal relevance would stipulate that simultaneous and backward causation are impossible on mere conceptual grounds. Yet, while simultaneous causation seems to be a physical possibility, backward causation, if impossible at all, is thought to be excluded on physical grounds. Resorting to manipulation, on the other hand, relativizes the non-symmetry of general causation to human intervention, where, intuitively, this non-symmetry seems to be perfectly independent of human existence. Causal processes - as planetary movements

\footnotetext{
${ }^{12}$ Cf. Eells (1991), Chap. 5, Hausman (1998), pp. 44-47.

${ }^{13}$ Cf. e.g. Armstrong (1983), Chap. 2.

${ }^{14}$ Cf. e.g. Suppes (1970) or Price (1992). Temporal order, of course, is not only non-symmetric in the above sense, but moreover asymmetric.

${ }^{15}$ Cf. e.g. Reichenbach (1956).

Springer
} 
or volcanic eruptions on Saturn - that are not manipulable by humans are non-symmetric and can be oriented just as everyday earthly processes as the breaking of a window or the starting of a car engine which are open to human intervention. Moreover, it is unclear how the notions of agency, intervention, and manipulation could be clarified without recourse to causation. In fact, these notions seem to straight-out presuppose clarity on causation. ${ }^{16}$

This is a generalizable consequence of implementing any external nonsymmetry for a theoretical account of the non-symmetry of general causation: The external non-symmetry becomes more basic than causal relevance. Thereby a straightforward causal analysis of these external non-symmetries is blocked. As long as we are not inevitably constrained to an analysis of the direction of causal relevance by means of an external non-symmetry, theoretical prudence calls for abstinence from recourse to such non-symmetries. Indeed, regularity theories are capable of capturing the direction of general causation without recourse to non-symmetries that are external to the conceptual framework of a regularity theoretic analysans of causal relevance.

General causation can be oriented on mere logical grounds. Roughly, while conditional dependencies among single factors cannot be attributed a direction without resorting to external non-symmetries, complex nets of such dependencies can be oriented based on existing regularities only. There are several alternative causes for each effect. A match can be lit by either striking it against a match box, by exposing it to fire or to a flammable chemical etc. Accordingly, causally interpretable regularities are far more complex than expressed by (III). Rather than merely one minimally sufficient condition, many alternative minimally sufficient conditions must be invoked for each effect. Moreover, instances of effects do not occur without at least one of their sufficient causes being instantiated. These mutual dependencies among causes and effects are tentatively expressible by means of a biconditional as in Eq. $4 .{ }^{17}$

$$
A C D \vee E F G \vee H I J \leftrightarrow B
$$

Each complex cause of $B$ is minimally sufficient for $B$, while the disjunction of all alternative causes is necessary for $B .{ }^{18}$ Ordinarily, only a small subset of the alternative causes involved in an investigated causal structure are known or of interest to that investigation. Below, variables $Y_{A_{1}}, Y_{A_{2}}$, etc. will be used to run over unknown or neglected elements of alternative causes of effects $A_{1}$ and $A_{2}$, respectively, but, for simplicity, let us here assume that $A C D, E F G$, and $H I J$ constitute all alternative causes of $B$.

\footnotetext{
${ }^{16}$ Cf. Woodward (2003), Chap. 3.

${ }^{17}$ Equation 4 is a mere tentative formal representation, for, as mentioned in "Hume's Legacy," propositional logic does not allow for adequately expressing the relational constraints implicit in causal regularities in the sense of (III) (cf. "First-Order Formalization" for details on the first-order representation of these constraints).

${ }^{18}$ This essentially corresponds to Mackie's (1974) famous analysis of causation in terms of so-called INUS-conditions. Mackie (1974) will not be given an in-depth review in the present context. This has been done in Baumgartner and Graßhoff (2004), Chap. 5.
} 
Equation 4 is non-symmetric with respect to the factors to the left and the right of " $\leftrightarrow$ ". The instantiation of a particular disjunct is minimally sufficient for $B$, but not vice versa. $B$ does not determine a particular disjunct to be instantiated. ${ }^{19} B$ only determines the whole disjunction of minimally sufficient conditions. Hence, given that an instantiation of $A C D$ is observed, it can be inferred that there is an instance of $B$ somewhere in the corresponding spatiotemporal neighborhood. On the other hand, if an instance of $B$ is observed, no such inference to a proximate instantiation of $A C D$ is possible. The observed instance of $B$ might well have been caused by $E F G$. This nonsymmetry corresponds to the non-symmetry of determination. It induces a specification of (III) along the following lines:

(IV) $A$ is causally relevant to $B$ iff the following conditions hold:

(1) $A$ is a part of a minimally sufficient condition $A X_{1}$ of $B$,

(2) $A X_{1}$ is a disjunct contained in a disjunction $A X_{1} \vee X_{2} \vee \ldots \vee X_{n}$, $n \geq 2$, of other minimally sufficient conditions of $B$, such that $A X_{1} \vee X_{2} \vee \ldots \vee X_{n}$ is necessary for $B$,

(3) The instances of $A X_{1} \vee X_{2} \vee \ldots \vee X_{n}$ and $B$ differ and are spatiotemporally proximate,

(4) There is an instance of $A X_{1}, X_{2}, \ldots$, and of $X_{n}$.

Clearly though, by contraposition Eq. 4 is equivalent to

$$
\bar{B} \leftrightarrow \neg(A C D) \wedge \neg(E F G) \wedge \neg(H I J)
$$

However, in view of the fact that effects have several alternative causes, (IV) restricts the causal interpretability of complex regularity statements to one specific syntactical form. Within a set of logically equivalent regularity statements, only expressions with a syntax that exhibits alternative minimally sufficient conditions as disjuncts of a necessary condition are causally interpretable. Applied to Eqs. 4 and 5, this syntactical constraint prohibits a causal interpretation of Eq. 5, for it does not render an underlying causal structure transparent in the sense just delineated. ${ }^{20}$

Equations 4 and 5 are moreover equivalent to a biconditional that results from Eq. 5 by factoring out and bringing the right-hand side back into disjunctive normal form:

$$
\overline{A E H} \vee \overline{A E I} \vee \ldots \vee \overline{D G J} \leftrightarrow \bar{B}
$$

In contrast to Eq. 5, Eq. 6 is unproblematically causally interpretable. While Eq. 4 identifies three minimally sufficient conditions as complex causes of $B$,

\footnotetext{
${ }^{19}$ Cf. Graßhoff and May (2001), pp. 97-99. Similar analyses of the direction of causation have been proposed in Russell (1913), p. 199, Sanford (1976) or Hausman (1998).

${ }^{20}$ The fact that logically equivalent expressions differ with respect to the straightforwardness of their causal interpretation is analogous to the fact that divergent normal forms differ with respect to how transparent they render truth conditions of logical formulae. For instance, it is much more intricate to read off truth conditions from prenex normal forms than, say, from normal forms with minimal quantifier scopes (cf. Hintikka 1973).
} 
Eq. 6 establishes the causally interpretable minimally sufficient conditions of $\bar{B}$. Each of those conditions amounts to a conjunction consisting of the negation of exactly one conjunct of each disjunct of Eq. 4. Furthermore, Eq. 6 does not reverse the direction of the dependencies expressed in Eq. 4. Both identify $B$ and $\bar{B}$, respectively, as effects and the other factors as causes. Thus, there is one regularity statement complying to the syntactical constraints imposed by (IV) for a positive effect and one for the latter's negative complement. Both of these regularities exhibit the same non-symmetry. Accordingly, neither of them poses a problem for (IV).

It might be objected that, while effects have multiple alternative causes, causes likewise could have multiple alternative effects. That means the following biconditional could be argued to describe a causal structure as well:

$$
A C D \vee E F G \vee H I J \leftrightarrow B_{1} \vee B_{2}
$$

Apparently, if a causal interpretation of Eq. 7 is in fact warranted, cause and effect factors cannot be discriminated by means of the non-symmetry of determination resorted to in (IV). Equation 7 is perfectly symmetric as regards determination of one side of the biconditional by the other: No factors on either side determine any other factors. For example, according to Eq. $7 A C D$ can be instantiated in combination with $B_{1} \overline{B_{2}}$ or with $\overline{B_{1}} B_{2}$. The analogue holds for, say, $B_{1}$ which is claimed to occur with either $A C D$ or $E F G$ or $H I J$ by Eq. 7. If Eq. 7 is causally interpreted, the principle of determinism is violated, for it is then no longer guaranteed that causes are always accompanied by the same effects. Equation 7 can only be seen to represent a causal structure if it is implicitly presupposed that causes are sometimes accompanied by a first effect and sometimes by a second. Indeed, a causal structure as stipulated by a causal interpretation of Eq. 7 could not be oriented in the vein of (IV). As to (IV) it is completely indeterminate which side of the biconditional in Eq. 7 is seen to contain causes and effects, respectively. As indicated in the introductory section, however, regularity theories presuppose causation to be a deterministic relation. Against the background of this presupposition, a causal interpretation of Eq. 7 is not warranted in the first place. Only biconditionals not featuring disjunctions on both sides can be seen to represent deterministic causal structures.

A successful discrimination between causes and effects in the vein of (IV) presupposes the validity of the principle of determinism. The vast majority of theories of causation indeed take causation to be deterministic. For, even though the existence of irreducibly indeterministic processes is hardly challengeable according to standard interpretations of quantum mechanics, there are many open questions - as for instance raised by phenomena of the EPR type $^{21}$ - with respect to the causal interpretability of these processes. For lack of space, the metaphysical question as to the validity of the principle of determinism cannot be properly addressed here. All that matters for our

${ }^{21}$ Cf. van Fraassen (1989). 
purposes is that symmetric biconditionals as Eq. 7 cannot be seen to represent deterministic causal structures and that, accordingly, the direction of a deterministic causal relation can be successfully accounted for by drawing on the non-symmetry of determination between cause and effect factors. If it should turn out that there are irreducibly indeterministic causal processes after all, the non-symmetry of such processes would have to be analyzed along different lines.

Accounting for the non-symmetry of general causation in the vein of (IV) has an important implication as regards the minimal complexity of causal structures. A factor or conjunction of factors $X_{1}$ that is both minimally sufficient and necessary for another factor or conjunction of factors $X_{2}$ cannot be identified as cause of $X_{2}$, for, in that case, $X_{2}$ is minimally sufficient and necessary for $X_{1}$ as well. All empirical evidence such a dependency structure generates is constituted by perfectly correlated instantiations of $X_{1}$ and $X_{2}-$ both are either co-instantiated or absent. Such empirical data is not causally interpretable. In order to distinguish causes from effects and to orient the cause-effect relation, at least two alternative causes are needed for each effect. It must be emphasized, however, that, while generic causal structures can be uncovered only based on data that meets these minimal diversity constraints, no such requirements must be satisfied in order to detect causal dependencies on token level. Normally, effect events are not overdetermined. Hence, in their particular situations of occurrence cause events are both sufficient and necessary for their effect events. Token causes and effects cannot be discriminated by means of the non-symmetry of determination. Instead, they can be kept apart by drawing on the generic causal structures they instantiate. Once the non-symmetry of general causation is accounted for, a token event $a$ can be identified as a cause of an event $b$ if $a$ and $b$ instantiate two factors $A$ and $B$, respectively, such that $A$ is a type level cause of $B .^{22}$

Contrary to the widespread opinion in the literature, regularity theories are not only capable of adequately capturing the non-symmetry of general causation, but moreover offer the prospect of successfully doing so without resorting to non-symmetries external to the conceptual framework implemented in their analysans of causation. Against this background, such external nonsymmetries as the direction of time or of human intervention remain amenable to a straightforward analysis in terms of the direction of causal relevance.

\section{Spurious Regularities}

One of the most widespread criticisms against regularity theories stems from so-called spurious regularities. ${ }^{23}$ Consider two parallel effects $A$ and $B$ of a common cause $C$ and assume, for simplicity's sake, that $C$ in fact is minimally

\footnotetext{
${ }^{22}$ This only indicates the general idea behind a regularity theoretic analysis of singular causation. For details see "Singular Causation."

${ }^{23}$ Cf. e.g. Cartwright (1989), pp. 25-29.

基 Springer
} 
Fig. 1 A common cause structure that gives rise to spurious regularities

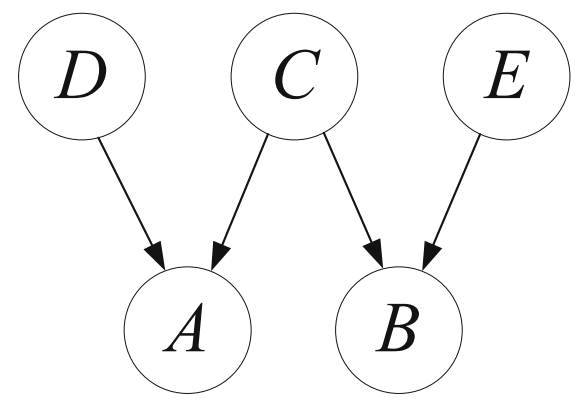

sufficient for $A$ and $B$. Let us suppose, furthermore, that there exists one minimally sufficient alternative cause for $A$ and $B$ each $-D$ for $A$ and $E$ for $B$. All in all, the causal structure under consideration thus is assumed to be of a form as depicted in Fig. 1. In this constellation, $A$ in combination with the absence of $D$, i.e. $A \bar{D}$, is minimally sufficient for $B$ without $A \bar{D}$ being a complex cause of $B$. Whenever $A \bar{D}$ occurs, $C$ is present as well, for no effect occurs without any of its causes. Hence, if $D$ is absent, $C$ must be present to account for $A$. Furthermore, since $C$ is taken to be sufficient for $B$, it follows that $A \bar{D}$ is sufficient for $B$ as well. Of course, $A \bar{D}$ is moreover part of a necessary condition of $B$ :

$$
A \bar{D} \vee C \vee E \leftrightarrow B
$$

According to (IV), Eq. 8 is a regularity statement that is causally interpretable. This clearly is an unacceptable consequence, for, as mentioned above, relative to the construction of the structure in Fig. $1, A \bar{D}$ is not causally relevant to $B$.

Structures as the one under consideration are ubiquitous. The most famous concrete example of this type is the so-called Manchester-Factory-Hooters example based on which Mackie (1974) ultimately abandoned the attempt to provide a genuine regularity theoretic analysis of general causation. ${ }^{24}$ Examples of this type unmistakably demonstrate that necessary conditions, just as sufficient conditions, may contain redundant elements. $A$ being necessary for $B$ implies that $A \vee C$ is necessary for $B$. Or formally:

$$
B \rightarrow A \vdash B \rightarrow A \vee C
$$

Any true conditional stays true if any (true or false) disjunct is added to its consequent. In analogy to the case of sufficient conditions, the extendability of necessary conditions by arbitrary disjuncts forecloses a causal interpretability of necessary conditions. A causal interpretation of necessary conditions is only warranted if the conditions exclusively contain factors that are essential to the bringing about of the purported effect. Arbitrary factors as $C$ in Eq. 9 or

\footnotetext{
${ }^{24}$ Cf. Mackie (1974), pp. 83-87, Cartwright (1989), pp. 25-29. In Baumgartner and Graßhoff (2004), pp. 99-103, we discuss the Manchester-Hooters case in all detail along with a solution to the problem it poses.
} 
conditions as $A \bar{D}$ in Eq. 8 must - even if they are minimally sufficient - not be incorporated in necessary conditions that are causally interpreted.

A solution to this problem that is parallel to the solution of the difficulties induced by monotony as been proposed in Graßhoff and May (2001): Necessary conditions must be minimalized. The latter are minimalized analogously to sufficient conditions: A necessary condition is minimally necessary iff it does not contain a necessary proper part. Minimalizing necessary conditions in this vein eliminates just the spurious minimally sufficient conditions as $A \bar{D}$ from complex regularity statements as Eq. 8. In order to see this, consider again the structure depicted in Fig. 1: Whenever $B$ is given, $C$ or $E$ is instantiated. Thus, $C \vee E$ is necessary for $B$. The antecedent of Eq. 8 has no other necessary proper part. $A \bar{D} \vee C$ is not necessary, for there are instances of $B$ without $A \bar{D}$ and $C$ being instantiated - say, when $A D$ is given along with $\bar{C}$ and $E$. Neither is $A \bar{D} \vee E$ necessary for $B$ : There are instances of $B$ without instances of $A \bar{D}$ and $E$ occurring - for example, when $A D$ is given in combination with $\bar{E}$ and $C$. Among the elements of the necessary condition of $B$ mentioned in Eq. 8 the following non-symmetry holds, which allows for eliminating $A \bar{D}: A \bar{D}$ is sufficient for $C \vee E$, while $C \vee E$ is not sufficient for $A \bar{D}$. That means $C \vee E$ is a minimally necessary disjunction of minimally sufficient conditions of $B$.

Reflecting this maximal minimalization, minimally necessary disjunctions of minimally sufficient conditions of a given factor are labeled minimal theories of that factor. A minimal theory of a factor $B$ is a minimally necessary disjunction $\phi$ of minimally sufficient conditions of $B$, such that (a) $\phi$ contains at least two disjuncts, (b) conjuncts in each disjunct are coincidently instantiated, (c) $B$ is instantiated in the spatiotemporal neighborhood of its minimally sufficient conditions, and (d) the instances of $B$ differ from the instances of its minimally sufficient conditions. The operator " $\Rightarrow$ " is introduced to abbreviate the formal expression corresponding to minimal theories. Accordingly, the minimal theory describing the behavior of $B$ in the exemplary structure depicted in Fig. 1 has the following form: ${ }^{25}$

$$
C \vee E \Rightarrow B
$$

$C \vee E$ is the antecedent of the minimal theory (10) and $B$ its consequent. A factor $Z$ is said to be part of a minimal theory $\Phi$ iff $Z$ is a conjunct of at least one disjunct in the antecedent of $\Phi$.

The notion of a minimal theory, however, is not itself sufficient to solve the problem posed by spurious regularities. For a proper minimalization of necessary conditions is always dependent on the availability of the proper minimally sufficient conditions involved in an investigated causal structure. This availability may not be guaranteed at the beginning of a causal investigation. Analyses of causal structures must be relativized to the set of examined factors, which shall be referred to as the factor frame of the analysis. Ordinarily, factor frames are gradually expanded in the course of investigating a structure.

\footnotetext{
${ }^{25}$ Cf. Baumgartner and Graßhoff (2004), Chap. 5. For details on the first-order form of minimal theories see "Minimal Theory" below.
} 
Suppose the scientific discipline investigating the causal structure depicted in Fig. 1 first discovers that $A \bar{D}$ and $E$ are minimally sufficient for $B$. At the same time, the scientists concerned with $B$ are confronted with instances of $B$ in situations where both $A \bar{D}$ and $E$ are absent. We who know the actual structure behind $B$, of course, can easily account for these cases: They are cases in which $D, C, B, \bar{E}$, and $A$ are instantiated. At this stage of scientific knowledge, however, the corresponding discipline will conjecture the validity of the following minimal theory featuring the variable $Y_{B}$ which runs over the unknown alternative causes of $B::^{26}$

$$
A \bar{D} \vee E \vee Y_{B} \Rightarrow B
$$

After a while of further investigation it is discovered that the formerly unknown factor $C$ constitutes an additional minimally sufficient condition of $B$, i.e. the factor frame of the causal analysis at hand is extended. Moreover, now the scientists can account for all instances of $B$. That means whenever $B$ is instantiated, there is an instance of $A \bar{D}, C$, or $E$. Thus, a necessary condition of $B$ has been discovered. This finding immediately raises the question as to whether this necessary condition is minimal. A disjunctive integration of $C$ into the antecedent of Eq. 11 yields:

$$
A \bar{D} \vee C \vee E
$$

As we have seen above, Eq. 12 is not a minimally necessary condition of $B$. Upon the discovery of $C, A \bar{D}$ becomes redundant and accordingly drops out of a minimalized necessary condition. Hence, extensions of factor frames can directly affect the membership of a condition in a minimal theory. The fact that $A \bar{D}$ is part of Eq. 11 does not guarantee that $A \bar{D}$ remains a constituent of every minimal theory resulting from extending the factor frame of Eq. 11. Therefore, despite all their causally interpretable features, minimal theories are not directly causally interpretable. A minimal theory is causally interpretable only if its factors stay part of that minimal theory across all extensions of the corresponding factor frame.

The fact that the causal interpretability of a minimal theory cannot be determined before its factor frame is fully expanded raises the question as to the status of minimal theories prior to a complete expansion of their factor frames. May (1999), p. 74, has pointed to an important feature of redundant minimally sufficient conditions as $A \bar{D}$ that allows for their identification prior to an exhaustive expansion of respective factor frames. Such redundant conditions contain causally dependent factors that cannot be absent at the same time, i.e. factors that are subcontrary. $A$ and $\bar{D}$ are causally dependent in the structure of Fig. 1. Against the background of that structure, these two factors cannot both be absent. If $A$ is not instantiated $(\bar{A})$, there cannot be an event of type $D$, thus $\bar{D}$ cannot be absent, and if $\bar{D}$ is not instantiated, i.e. if $D$ is present, $A$ cannot be absent. Such causal dependencies among factors of minimally

${ }^{26}$ Cf. "Minimal Theory" for detailed introduction of $Y_{X}$-variables. 
sufficient conditions are 'minimalized away' as respective factor frames are extended.

Apart from a relativization of causal reasoning to a given factor frame the minimalization of necessary conditions induced by the problem of spurious regularities calls for a distinction between direct and indirect causal relevance. For all indirect causes of an effect as $B$ in Fig. 1 are redundant in a minimally necessary condition of $B$. Nonetheless, indirect causes are causally relevant to their indirect effects. Suppose, we introduce two alternative direct causes of $C$ into the structure of Fig. 1: $F$ and $G$ shall each be minimally sufficient for $C$. As direct causes of $C, F$ and $G$ are indirect causes of $B$. However, Eq. 10 does not allow for an integration of further factors. Both $F$ and $G$ imply $C \vee E$ while the converse does not hold, thus, both $F$ and $G$ are redundant in a necessary condition of $B$ featuring $C \vee E$.

Yet, even though causal relevancies cannot be directly read off minimal theories, the latter constitute the core of the conceptual inventory needed for a successful regularity theoretic handling of spurious regularities. While membership in minimal theories that resists factor frame extensions accounts for direct causal relevance, the transitive closure thereof accounts for indirect relevance:

(V) (a) $A$ is directly causally relevant to $B$ iff the following conditions hold:

(1) $A$ is a part of a minimal theory $\Phi$ of $B$,

(2) $A$ stays part of $\Phi$ across all extensions of $\Phi$ 's factor frame.

(b) $A$ is indirectly causally relevant to $B$ iff there is a sequence of factors $Z_{1}, Z_{2}, \ldots, Z_{n}, n \geq 3$, such that $A=Z_{1}, B=Z_{n}$, and for each $i$, $1 \leq i<n: Z_{i}$ is directly causally relevant to $Z_{i+1}$ in terms of (Va).

As May (1999), pp. 67-68, has shown, introducing a minimality constraint on necessary conditions has a very important existential implication that allows for dropping condition (4) of (IV): $C \vee E$ being minimally necessary for $B$ implies there being an instance of $B$ without a corresponding instance of $C$ refer to this scenario as $S_{1}$ - and an instance of $B$ without a corresponding instance of $E$-scenario $S_{2}$. That is, none of the two disjuncts is itself necessary for $B$. Nonetheless the disjunction as a whole is necessary for $B$. Therefore, both in $S_{1}$ and in $S_{2}$ there must be an instance of $C \vee E$. Since in $S_{1}$, by assumption, there is no event of type $C$, there must be an event of type $E$ - and vice versa for $S_{2}$. This finding is generalizable: Every disjunct of a minimally necessary condition is instantiated at least once along with the corresponding effect, when all the other disjuncts are absent.

\section{Single-Case and Other Accidental Regularities}

The problem induced by spurious regularities is not the only objection raised against regularity theories of type (IV) in the literature. A problem for (IV) that is closely related to the problem of empty regularities has e.g. been raised 
by Armstrong (1983). ${ }^{27}$ A conditional turns out true if both its antecedent and consequent are true. Thus, if antecedent and consequent of a conditional each report the occurrence of a singular event that actually has occurred, the conditional as a whole is true. Therefore, Armstrong argues, a regularity as required by a regularity theory exists among any two factors with a single instance each, irrespective of whether they are causally related or not. No doubt, a conditional as "Whenever Nero sets fire on Rome, the Titanic sinks" is true and, no doubt, we are not prepared to hold Nero causally responsible for the sinking of the Titanic. Hence, Armstrong's argument continues, not only empty, but also these so-called single-case regularities pose a serious problem for a regularity theory.

At first, it must be pointed out that the plain truth of a conditional as "Whenever Nero sets fire on Rome, the Titanic sinks" does not suffice to identify Nero's setting fire on Rome as a cause of the sinking of the Titanic according to any of the regularity theoretic accounts (I) to (V) considered thus far. For these accounts not only require causes and effects to satisfy a material conditional, but moreover to be proximately instantiated. More is said about spatiotemporal proximity in "Spatiotemporal Proximity of Causes and Effects." However, relative to any pre-theoretic understanding of that notion it seems plain that Nero's setting fire on Rome and the sinking of the Titanic cannot be seen as proximate events. This shortcoming of Armstrong's argument is easily remedied. Assume that Harold Bride, the junior wireless operator on the Titanic, for the first (and only) time in his life lit a Havana cigar moments before the ship hit the iceberg. The conditional "Whenever Harold Bride lights a Havana, the Titanic sinks" is true and, moreover, the instances of its antecedent and consequent are spatiotemporally proximate. Of course, Bride's lighting of a Havana is not only sufficient, but moreover minimally sufficient for the Titanic to sink. The antecedent of the above regularity statement does not comprise a sufficient proper part. Furthermore, Bride's lighting of a Havana is not the only minimally sufficient condition of the sinking of the Titanic. The latter's real cause constituted by the collision with the iceberg amounts to another such condition. Hence, there is a necessary condition of the sinking of the Titanic that contains Bride's lighting of a Havana as a minimally sufficient disjunct. This suffices to refine Armstrong's argument such that it does justice to the complexity of causal structures as required by (IV): Any two factors with exactly one instance each, such that these instances are spatiotemporally proximate, satisfy a regularity as required by (IV), yet by no means all thus related factors are related in terms of causal relevance as well. Consequently, (IV) does not amount to a sufficient condition for causal relevance.

That (IV) is unsuited as analysans of causal relevance has already been demonstrated by the problem of spurious regularities. In order for Armstrong to succeed in establishing that single-case regularities prove the fundamental defectiveness of regularity accounts, his argument must be directed against

${ }^{27}$ Cf. Armstrong (1983), pp. 15-17, similarly Mellor (1995), p. 15, or Hausman (1998), p. 42. 
(V). It must be shown that Bride's lighting of a Havana is not only contained in a necessary condition of the sinking of the Titanic, but moreover is a non-redundant part of a causally interpretable minimally necessary condition thereof, i.e. that it is part of a minimal theory of the Titanic's sinking. Furthermore, it must be shown that Bride's lighting of a Havana stays part of that minimal theory across all factor frame extensions.

Before this further refinement will be attempted a possible objection against Armstrong's argument has to be considered. Antecedent and consequent of "Whenever Harold Bride lights a Havana cigar, the Titanic sinks" involve proper names or, if formal explications by means of definite descriptions are preferred, predicates that apply to single events only - more generally: local predicates, i.e. predicates that involve spacetime coordinates or singular terms. The admissibility of local predicates in contexts of general causation, as is well known, is commonly denied in the literature. The regularity under consideration here shall therefore be rephrased in non-local terms. Let us grant that "Whenever Harold Bride lights a Havana cigar, the Titanic sinks" represents the exact same single-case regularity as "Whenever a person with genome $s$ lights a Havana cigar, an ocean liner with molecular structure $t$ sinks", or formally $S \rightarrow T$.

The Titanic's collision with the iceberg, of course, is expressible by means of non-local predicates as well. Symbolizing this (non-locally defined) collision by $C$ we get the following true biconditional that is causally interpretable according to (IV):

$$
S \vee C \leftrightarrow T
$$

If $S \vee C$ is not merely necessary, but moreover both minimally necessary for $T$ and resistant against factor frame extensions, Eq. 13 not only refutes (IV), but also (V). $S \vee C$, however, does not amount to a minimally necessary condition of $T$. There is only one single instance of each factor involved in Eq. 13. Whenever $T$ occurs, both $S$ and $C$ are present nearby. Thus, the antecedent of Eq. 13 can be further minimalized:

$$
\begin{aligned}
& S \leftrightarrow T \\
& C \leftrightarrow T
\end{aligned}
$$

Neither Eqs. 14 nor 15, however, correspond to minimal theories, for these expressions merely report a perfect correlation of $T$ and $S$ and $C$. Any of these factors is given if and only if the other two factors are given as well. Such perfect correlations, as we have seen above, are not causally interpretable, for none of the involved factors is identifiable as cause and effect, respectively. Since (V) requires causally interpretable regularities to specify minimally necessary conditions of a certain minimal complexity, neither Eqs. 14 nor 15 is amenable to a causal interpretation according to (V). That, however, does not mean that $(\mathrm{V})$ does not allow for identifying the collision with the iceberg as a cause of the sinking of the Titanic. The impossibility to causally interpret either Eqs. 14 or 15 merely indicates that the events involved in the sinking of the Titanic are typecast in an overly fine-grained manner. Causal structures 
can only be unfolded when a sufficient amount of comparable test situations are available. Thus, the Titanic's sinking must be placed in a broader context, it must be typecast in a more coarse-grained fashion, e.g. as a sinking of an ocean liner. Such a typing will immediately yield far more instances for each causal factor, which, in turn, will suspend biconditional dependencies as in Eqs. 14 or 15. If not only the Titanic's sinking, but ocean liner sinkings are taken into consideration, many cigar lightings will be found that are in no way followed by a sinking ocean liner, whereas collisions with icebergs either retain their sufficiency for ocean liner sinkings or a subsequent expansion of the corresponding factor frame will reveal them to be parts of minimally sufficient conditions of such sinkings. Even within an extended factor frame, cigar lightings, on the other hand, will not turn out to be non-redundant parts of sufficient conditions of ocean liner sinkings.

As mentioned before, a regularity theoretic analysis of causal relevance along the lines of (V) imposes important minimal complexity constraints on generic causal structures. Generic structures are not one-to-one dependencies. Every effect has several alternative complex causes. In order to establish such dependencies, a certain amount of diversity in the empirical data is called for. Without such diversity no causal diagnosis is possible. The successful discovery of causal structures within any available theory of causation essentially hinges on the quality of the available data. The regularity theoretic notion of causal relevance expressed in (V) mirrors these minimal complexity requirements and, accordingly, is not affected by the problem of single-case regularities.

The smaller the set of instances of a factor, the higher the chances that accidental regularities as in the Titanic case exist. However, single-case regularities only constitute a special type of accidental regularities. Regularities that are not causally interpretable also appear in many-case scenarios. In order to consider whether accidental regularities of the many-case type pose a problem for (V), let us somewhat modify the shipwreck example. Suppose Shamus and Fennella both are proud owners of a sailboat. Moreover, independently of each other they both are hit by a storm, factor $H$, while sailing out in the lake, to the effect that both of their boats sink. To the sinking of a sailboat we shall refer by $W$. During the storm they both stumble, are thrown against the railings of their boats and lose their left maxillary central incisors. Shamus' mishap shall be labeled $I$ and Fennella's $J$. As humans can lose specific incisors only once, the following regularities hold for our two exemplary shipwrecks: Whenever Shamus or Fennella lose their left maxillary central incisors, there is a sinking of a sailboat, or formally

$$
I \vee J \rightarrow W
$$

The converse, i.e. $W \rightarrow I \vee J$, does not hold. There are many sinkings of sailboats without instances of either $I$ or $J$. Thus, this example does not feature biconditional dependencies as in the Titanic case. Moreover, as there are two alternative minimally sufficient conditions of $W$, the minimal complexity constraints imposed on causal structures by (V) are met. Does Eq. 16, hence, 
express accidental regularities that erroneously have to be causally interpreted according to $(\mathrm{V})$ ?

Equation 16 is not a minimal theory of $W$, for, as mentioned above, it does not cover all instances of $W$. Most sailboats sink when there is a storm without loss of teeth. Moreover, there are countless further alternative causes of shipwrecks, for instance fires or collisions with icebergs. If these further causes are all represented by the variable $Y_{W}$, we get the following biconditional dependency:

$$
I \vee J \vee H \vee Y_{W} \leftrightarrow W
$$

If Eq. 17 is a minimal theory involving factors that are resistant to factor frame extensions, Eq. 17 must be causally interpreted according to (V), which, as a consequence, would be seriously defective. Equation 17, however, is not a minimal theory of $W . I \vee J \vee H \vee Y_{W}$ is not minimally necessary for $W$; it contains a necessary proper part, viz. $H \vee Y_{W}$. Whenever either Shamus or Fennella lose their incisors there is a storm, thus $I \vee J \rightarrow H$. Due to this dependency $I$ and $J$ are redundant in necessary conditions containing $H$. That means the real causes of an effect render conditions, that are merely accidentally minimally sufficient, redundant in minimal theories. The minimalization of necessary conditions as demanded by (V) not only precludes spurious regularities from a causal interpretation but also accidental regularities. A regularity theory in the vein of $(\mathrm{V})$ is not forced to causally interpret accidental regularities of the many-case type.

\section{Singular Causation}

The problem posed by single-case regularities demonstrates that a regularity theory cannot be successful if its primary analysans is taken to be singular causation, i.e. causation among token events. Directly accounting for singular causation by means of sufficient and necessary conditions inevitably generates a host of accidental regularities whose causal interpretation can hardly be avoided without resorting to some form of nomic sufficiency or necessity and, thus, without leaving the uncontroversial path of first-order languages. In contrast, the previous sections have shown that the complexity of generic causal structures imposes important additional constraints on causal dependencies on type level which allow for distinguishing between accidental and causal regularities. Nonetheless, some regularity theories, as e.g. developed in Mackie (1965), aim to analyze singular causation by means of first-order sufficient and necessary conditions. In consequence, a lot of the criticism raised against regularity theories over the past four decades targets this kind of singularist account. In addition to their inability to avoid a causal interpretation of accidental regularities it has, for instance, been claimed that singularist regularity theories cannot properly deal with so-called preempted potential causes. ${ }^{28}$ As

\footnotetext{
${ }^{28}$ Cf. e.g. Collins et al. (2004).

Springer
} 
the regularity theory presented in this paper, however, focuses on general causation from the outset, the singularist thread in the literature has been neglected here.

Rather than discussing the problems encountered by an analysis of singular causation in terms of sufficient and necessary conditions, the argument of this paper shall be concluded by indicating how singular causation can be accounted for by drawing on a regularity theoretic account of general causation as given in (V). The basic idea behind the identification of token level causes and effects based on (V) is utterly straightforward and has already been anticipated in "Non-Symmetry." Instead of stipulating that an event $a$ is a cause of an event $b$ if $a$ is in some sense sufficient or necessary for $b$, (V) paves the way for identifying $a$ as a cause of $b$ if $a$ and $b$ instantiate two factors $A$ and $B$, respectively, such that $A$ is causally relevant to $B$. More specifically, in light of $(\mathrm{V})$ the following transition from general to singular causation is at hand:

Singular Causation (SC): An event $a$ is a cause of an event $b$ iff $a$ instantiates a factor $A$ and $b$ instantiates a factor $B$, such that

(a) $A$ is a non-redundant part of a minimally sufficient condition $A X$ of $B$ and every factor contained in $A X$ is causally relevant to $B$ according to $(\mathrm{V})$,

(b) $a \neq b$, and $a$ and $b$ occur within the same spatiotemporal frame,

(c) Every factor in $X$ is instantiated coincidently with $a$.

As (V), this account of singular causation presupposes the admittedly vague notions of a spatiotemporal frame and of coincidence. More is said about these notions in the Appendix. In order to see the main features and qualities of (SC), however, an intuitive assessment of when events are spatiotemporally proximate as opposed to distant will suffice. First, note that (SC) yields an irreflexive notion of singular causation. Cause and effect events are required to differ. While event types may be causally relevant to themselves, there is no self-causation on token level. Second, (SC) does not exclude token level feedbacks. If there is a causally interpretable minimal theory featuring $A$ as a cause of $B$ and another such theory featuring $B$ as a cause of $A, a$ may be determined to cause $b$ which, in turn, may be determined to cause $a$ by (SC). The existence of token level feedbacks is controversial in the literature. Candidates for such feedbacks are e.g. static equilibria. One of the most discussed examples of this kind is a stable house of cards. The two cards at the top lean against each other. Each prevents the other from falling over. ${ }^{29}$ The debate as to the causal nature of static equilibria shall not be entered into here. Whatever the outcome will be, it seems plain that the question as to whether the card example constitutes a case of token level feedback or not is not to be decided on a priori conceptual grounds. The existence of simultaneous reciprocal singular causation is to be determined synthetically.

${ }^{29}$ Cf. e.g. Fair (1979), p. 230, Frankel (1986), and Hausman (1998), pp. $44-47$. 
(SC), accordingly, does not rule out token level feedbacks. That means (SC) yields a non-symmetric, but not an asymmetric notion of singular causation.

Apart from being non-committal with respect to the existence of causal feedbacks on token level, (SC) has further notable merits. According to (SC), an event $a$ is only identified to cause an event $b$ if $a$ occurs along with all other events instantiating a minimally sufficient condition $A X$ of $B$. As a direct consequence of this constraint, preempted potential causes of $b$ are not identified as genuine causes of $b$ by (SC). Consider, for instance, the famous desert traveler one of whose enemies drills a hole into his drinking bottle and another poisons the water in the bottle. ${ }^{30}$ The traveler then dies of thirst. The poisoning of the water is preempted by the perforation of the bottle. (SC) indeed only identifies the latter event as a cause of the traveler's death, for crucial factors - as e.g. the drinking of the poisonous water - in combination with which poisoning water only becomes lethal are not instantiated in the desert traveler case.

Finally, reconsider the shipwreck of the Titanic. Even though Harold Bride's lighting of a Havana cigar, in the circumstances, is both sufficient and necessary for the sinking of the Titanic, (SC) does not determine Bride's action to cause the end of the Titanic. There is no causally interpretable minimal theory $\Phi$ such that Bride's cigar lighting instantiates the antecedent of $\Phi$ and the sinking of the Titanic instantiates $\Phi$ 's consequent. The accidental regularity between Shamus' loss of a tooth and his shipwreck is handled analogously. Shamus' tooth loss does not instantiate a factor which remains part of a minimal theory of shipwrecks across factor frame extensions. Contrary to a singularist regularity theory, thus, (SC) does not erroneously causally interpret accidental regularities among event tokens.

\section{Conclusion}

Empty, single-case, and other accidental regularities, common cause structures and the non-symmetry of general causation can all be adequately captured in regularity theoretic terms. Regularity theories can do justice to the whole complexity of generic causal structures. And all this is accomplished with simple recourse to extensional standard logic. Appropriate minimalization strategies are at hand such that redundancies implicit in material conditionals - e.g. due to monotony - can be efficaciously precluded from a causal interpretation. Characteristics of complex causal nets can be resorted to in order to distinguish causal from spurious and accidental regularities. Finally, we have seen that a regularity theoretic analysis of general causation can pave the way towards a straightforward account of singular causation. All in all, this paper should have shown that regularity theories can be conceived in a way that constitutes a promising and very intuitive alternative to popular theoretical frameworks as implemented by counterfactual or probabilistic analyses.

\footnotetext{
${ }^{30}$ Cf. Mackie (1974), pp. 44-46.

型 Springer
} 
Acknowledgements I would like to thank Timm Lampert and Gerd Graßhoff for valuable discussions about the issues addressed in this paper and three anonymous referees for this journal for very helpful comments on an earlier draft. Moreover, I am grateful to the Swiss National Science Foundation for generous support of this work (Grant 1114-066803.01/1).

\section{Appendix}

\section{First-Order Formalization}

This appendix introduces the first-order formalizations of the core notions involved in a regularity theory of type (V). As indicated in "Hume's Legacy," the reason for a first-order representation of causal regularities lies in the relational constraints that distinguish causally interesting regularities from causally meaningless regularities as "Whenever there is a table, there is a table". Causes and effects are instantiated by spatiotemporally proximate events, causal regularities are instantiated by different events, and factors constituting a complex cause are instantiated coincidently. These three relational constraints characterizing causal regularities must be accounted for by first-order means. I take them in turn.

\section{Spatiotemporal Proximity of Causes and Effects}

In accordance with Broad (1930), $\phi$ shall be defined to be a sufficient condition of $\psi$ iff Eq. 18 holds and a necessary condition of $\psi$ iff Eq. 19 holds:

$$
\begin{aligned}
& \forall \mu(\phi \mu \rightarrow \psi \mu) \\
& \forall \mu(\psi \mu \rightarrow \phi \mu)
\end{aligned}
$$

$\mu$ is to be read as a metavariable running over variables and $\phi \mu$ and $\psi \mu$ stand for any well-formed formulae with at least one free occurrence of $\mu$.

Instances of causes and instances of their effects do not occur anywhere and anytime, but close by, i.e. within a certain spatiotemporal frame or within the same situation. The interpretation of the relation "... occurs in the same spatiotemporal frame as ..." cannot be fixed to a certain spatiotemporal interval independently of a causal process under investigation. However, in order to make sure that no causal element is covertly introduced into the notion of spatiotemporal proximity the latter shall be determined to be a symmetric relation, which - as mentioned above - causal relevance clearly is not. Certain instances of causes and effects can be said to be properly related only if they are in direct spatiotemporal contact, while in other cases instances of causes may well occur far away from the instances of their effects. The theory of special relativity only provides an upper bound for this interval: Instances of causes and effects must occur within each other's light cones. Notwithstanding this lacking specificity, given a concrete causal process it is normally uncontroversial which events can be said to be properly related 
in order to be amenable to a causal interpretation. ${ }^{31}$ Moreover, whenever spatiotemporal proximity is unsuitably interpreted for a given causal process, no dependencies appear in corresponding empirical data. ${ }^{32}$

If minimally sufficient and minimally necessary conditions should, at least in principle, be open for causal interpretations, the syntax of acceptable substitutions in Eqs. 18 and 19 must be restricted such that an instance $x$ of a cause factor and an instance $y$ of an effect factor are required to occur in the same spatiotemporal frame. In order to formally represent this spatiotemporal association, we introduce the symmetric relation Rxy. This induces a first approximation to a first-order representation of a causal regularity. Whoever claims that $A$ is a (sufficient) cause of $B$, claims that for all events $x$ of type $A$ there is an event $y$ of type $B$ such that $x$ and $y$ occur in the same spatiotemporal frame. This is captured by Eq. 20.

$$
\forall x(A x \rightarrow \exists y(B y \wedge \mathrm{R} x y))
$$

\section{Causal vs. Semantic Regularities}

Equation 20 not only describes causal regularities, but also what might be referred to as semantic regularities or regularities of set inclusion as "Whenever there is a soccer game, there is a sport event". A semantic regularity is given in case of two predicates one of which has an extension that is included in the extension of the other. Accordingly, by satisfying the first of these predicates an object or event eo ipso satisfies the other predicate. As single objects or events are moreover spatiotemporally proximate to themselves, one and the same object or event satisfies antecedent and consequent of a semantic regularity. The soccer games and the sport events are identical, thus, they certainly are spatiotemporally proximate according to any spatiotemporal interval chosen as interpretation of $\mathrm{R}$. In order to exclude semantic regularities from consideration when it comes to causal analyses, it must be stipulated that instances of causes differ from the instances of their effects. No token event ever causes itself. Hence, Eq. 20 must be specified such that all models of the

\footnotetext{
${ }^{31}$ If the striking of a match in Switzerland is followed by a match catching fire in England, it is clear that, even if the interval between these two events is thus that they are not excluded to be causally related by special relativity, the striking in Switzerland does not cause the light in England. On the other hand, given a concrete run of an experiment, say a number of substances are brought together in a test tube, it is commonly presumed that no events occurring in the course of this run are excluded from a causal dependency due to inadequate spatiotemporal relatedness. Accordingly, whenever the spatiotemporal relation between singular causes and effects is explicitly discussed in theoretical accounts of causation, it is commonly left as unspecified as possible (cf. e.g. Xu 1997, pp. 159-160).

${ }^{32}$ As I show in Baumgartner (2006), Chap. 5, the relation "... occurs in the same spatiotemporal frame as ..." can be suitably interpreted by means of a kind of trial-error procedure for every causal context under investigation.
} 
specified formula feature different events as instances of the factors contained in antecedent and consequent, respectively:

$$
\forall x(A x \rightarrow \exists y(B y \wedge \mathrm{R} x y \wedge x \neq y))
$$

Equation 21 states that for all events $x$ of type $A$ there is a different event $y$ of type $B$ in the same spatiotemporal frame as $x$. In order to conveniently abbreviate our notation, we introduce “ $\mapsto$ ”:

$$
Z_{1} \mapsto Z_{2}={ }_{d f} \forall x\left(Z_{1} x \rightarrow \exists y\left(Z_{2} y \wedge x \neq y \wedge \mathrm{R} x y\right)\right)
$$

\section{Coincidence}

The factors of a complex cause only become causally effective if coincidently instantiated. Hence, the factors contained in a minimally sufficient condition must be required to be coincidently instantiated. In order to symbolically represent coincident instantiations, I introduce the $n$-ary relation $K$ with $n$ being the number of conjuncts in a minimally sufficient condition apart from $K$ itself. $K$ subsists among the instances of the factors in a minimally sufficient condition iff these factors are coincidently instantiated. $K$ can be seen on a par with any ordinary non-redundant factor within a minimally sufficient condition. If $K$ does not hold among the instances of a conjunction of factors, the instantiations of these factors are not sufficient for the effect to occur. Thus, in contrast to $\mathrm{R}, K$ may remain uninterpreted.

Only the subset of minimally sufficient conditions that include $K$ can possibly be causally interpreted. A possibly causally interpretable minimally sufficient condition is of the form:

$$
\begin{aligned}
\forall x_{1} \forall x_{2} \ldots \forall x_{n}\left(A_{1} x_{1} \wedge A_{2} x_{2} \wedge \ldots \wedge A_{n} x_{n} \wedge K x_{1} x_{2} \ldots x_{n}\right. \\
\left.\rightarrow \exists y\left(B y \wedge x_{1} \neq y \wedge x_{2} \neq y \wedge \ldots \wedge x_{n} \neq y \wedge \mathrm{R} x_{1} x_{2} \ldots x_{n} y\right)\right)
\end{aligned}
$$

As Eq. 23 demonstrates, the factors of a possibly causally interpretable minimally sufficient condition are not required to be instantiated by the same event. As long as they occur coincidently, factors in a complex cause may well be instantiated by different events. The complexity of Eq. 23, which describes a minimally sufficient condition by explicitly mentioning three factors only, apparently calls for further abbreviations. To this end we adopt the convention that a conjunction of factors whose instances are related in terms of $K$ shall simply be concatenated without conjunctor and without explicit mention of $K$. A universally or existentially quantified conjunction of factors $A_{1} x_{1} \wedge$ $A_{2} x_{2} \wedge \ldots \wedge A_{n} x_{n} \wedge K x_{1} x_{2} \ldots x_{n}$, accordingly, is represented by $A_{1} A_{2} \ldots A_{n}$.

$$
A_{1} A_{2} \ldots A_{n}={ }_{d f} A_{1} x_{1} \wedge A_{2} x_{2} \wedge \ldots \wedge A_{n} x_{n} \wedge K x_{1} x_{2} \ldots x_{n}
$$

The quantifiers that bind the variables on the right-hand side of Eq. 24 can be left unspecified, because this abbreviated notation is only used in connection with “ $\mapsto$ ", whose antecedent is determined to be universally quantified and 
whose consequent is existentially quantified by definition. Therefore, the context in which expressions of type $A_{1} A_{2} \ldots A_{n}$ appear always clarifies the nature of the quantifiers involved. Given this notational convention, Eq. 23 can be transparently stated thus:

$$
A_{1} A_{2} \ldots A_{n} \mapsto B
$$

\section{Minimal Theory}

Before minimal theories can be formally represented, the abbreviated notation initiated in the previous sections needs to be extended. Minimally sufficient and minimally necessary conditions have been defined as (finite) open conjunctions and disjunctions. In order to account for that openness, two types of variables running over factors shall be introduced. For conjunctions of unknown or unspecified factors within sufficient conditions we shall implement the variables $X_{1}, X_{2}$, etc. Thus, these $X$-variables are to be read as running over factors that are not explicitly integrated within sufficient conditions.

$$
X_{i}={ }_{d f} Z_{1} x_{1} \wedge Z_{2} x_{2} \wedge Z_{3} x_{3} \wedge \ldots \wedge Z_{n} x_{n}, n \geq 1
$$

with $i=1,2,3, \ldots$, the variables $Z_{1} \ldots Z_{n}$ running over factors, and quantification depending on whether $X_{i}$ appears right or left of “ $\mapsto$ ”.

Building on the definition of $X_{i}$, we define the variables $Y_{A}, Y_{B}$, etc. to represent disjunctions whose disjuncts are not explicitly integrated within necessary conditions. The subscripts in case of the $Y$-variables correspond to the factors whose necessary condition a respective $Y$-variable complements.

$$
Y_{x}={ }_{d f} X_{1} \vee X_{2} \vee X_{3} \vee \ldots \vee X_{n}, n \geq 1,
$$

with $x=A, B, C, \ldots$ and quantification equally depending on whether $Y_{x}$ appears right or left of “ $\mapsto$ ”.

With these notational means at hand, a factor $A$ being part of a minimally sufficient condition of $B$, such that this minimally sufficient condition, in turn, is part of a minimally necessary condition of $B$ can be expressed thus:

$$
\left(A X_{1} \vee Y_{B} \mapsto B\right) \wedge\left(B \mapsto A X_{1} \vee Y_{B}\right)
$$

Equation 25 states that whenever $A$ is instantiated coincidently with other factors $X_{1}$ or one of the disjuncts in the domain of $Y_{B}$ is instantiated, the factor $B$ is instantiated in the same spatiotemporal frame by an event that differs from the instances of $A X_{1} \vee Y_{B}$; and whenever $B$ is instantiated, there is either a coincident instantiation of $A X_{1}$ or one of the disjuncts in the domain of $Y_{B}$ is instantiated in the same spatiotemporal frame, such that the instances of $B$ and of $A X_{1} \vee Y_{B}$ differ. Equation 25 can thus be seen as an abbreviation of expressions of the form of Eq. 26, where the incompleteness is indicated 
by dots instead of $X$ - and $Y$-variables and $k$ and $i$ stand for arbitrary natural numbers. ${ }^{33}$

$$
\begin{aligned}
& \forall x_{1} \ldots \forall x_{i}\left(\left(A_{1} x_{1} \wedge A_{2} x_{2} \wedge \ldots \wedge A_{i} x_{i} \wedge K x_{1} x_{2} \ldots x_{i}\right)\right. \\
&\left.\quad \rightarrow \exists y\left(B y \wedge x_{1} \neq y \wedge \ldots \wedge x_{i} \neq y \wedge \mathrm{R} x_{1} \ldots x_{i} y\right)\right) \\
& \quad \wedge \ldots \wedge \forall x_{1} \ldots \forall x_{k}\left(\left(Z_{1} x_{1} \wedge Z_{2} x_{2} \wedge \ldots \wedge Z_{k} x_{k} \wedge K x_{1} x_{2} \ldots x_{k}\right)\right. \\
&\left.\quad \rightarrow \exists y\left(B y \wedge x_{1} \neq y \wedge \ldots \wedge x_{k} \neq y \wedge \mathrm{R} x_{1} \ldots x_{k} y\right)\right) \\
& \quad \wedge \forall y\left(B y \rightarrow \left(\exists x _ { 1 } \ldots \exists x _ { i } \left(A_{1} x_{1} \wedge A_{2} x_{2} \wedge \ldots \wedge A_{i} x_{i} \wedge K x_{1} x_{2} \ldots x_{i}\right.\right.\right. \\
&\left.\quad \wedge x_{1} \neq y \wedge \ldots \wedge x_{i} \neq y \wedge \mathrm{R} x_{1} \ldots x_{i} y\right) \vee \ldots \vee \exists x_{1} \ldots \exists x_{k}\left(Z_{1} x_{1} \wedge Z_{2} x_{2}\right. \\
&\left.\left.\left.\wedge \ldots \wedge Z_{k} x_{k} \wedge K x_{1} x_{2} \ldots x_{k} \wedge x_{1} \neq y \wedge \ldots \wedge x_{k} \neq y \wedge \mathrm{R} x_{1} \ldots x_{k} y\right)\right)\right)
\end{aligned}
$$

Equation 26 clearly illustrates that minimal theories are highly intricate and non-transparent first-order expressions. In order to abbreviate our notation further, we introduce " $\Rightarrow$ ":

$$
Z_{1} \vee Z_{2} \Rightarrow Z_{3}={ }_{d f}\left(Z_{1} \vee Z_{2} \mapsto Z_{3}\right) \wedge\left(Z_{3} \mapsto Z_{1} \vee Z_{2}\right)
$$

This allows for transparently expressing Eqs. 25 and 26 as follows:

$$
A X_{1} \vee Y_{B} \Rightarrow B
$$

\section{References}

Armstrong, D. M. (1983). What is a law of nature? Cambridge: Cambridge University Press.

Baumgartner, M. (2006). Complex causal structures. Extensions of a regularity theory of causation. Ph.D. thesis, University of Bern, Bern.

Baumgartner, M., \& Graßhoff, G. (2004). Kausalität und kausales Schliessen. Eine Einführung mit interaktiven Übungen. Bern: Bern Studies.

Brand, M., \& Swain, M. (1970). On the analysis of causation. Synthese, 21, 222-227.

Broad, C. D. (1930). The principles of demonstrative induction I-II. Mind, 39, 302-317, 426-439.

Cartwright, N. (1989). Nature's capacities and their measurement. Oxford: Clarendon Press.

Collins, J., Hall, N., \& Paul, L. (Eds.) (2004). Causation and counterfactuals. Cambridge: MIT Press.

Davidson, D. (1980/1967). Causal relations. In Essays on actions and events (pp. 149-162). Oxford: Clarendon Press.

Eells, E. (1991). Probabilistic causality. Cambridge: Cambridge University Press.

Fair, D. (1979). Causation and the flow of energy. Erkenntnis, 14, 219-250.

\footnotetext{
${ }^{33}$ In Eq. 26 sufficient conditions are not disjunctively assembled as in Eq. 25. This divergence from Eq. 25 is warranted by the following logical equivalence that allows for a disjunctive assembling and a corresponding disintegration of sufficient conditions:

$$
\begin{aligned}
\forall x(A x \rightarrow \exists y(C y \wedge x \neq y \wedge \mathrm{R} x y)) \wedge \forall x(B x \rightarrow \exists y(C y \wedge x \neq y \wedge \mathrm{R} x y)) \\
\quad \mapsto \vdash \forall x(A x \vee B x \rightarrow \exists y(C y \wedge x \neq y \wedge \mathrm{R} x y))
\end{aligned}
$$
}

Or abbreviated:

$$
(A \mapsto C) \wedge(B \mapsto C) \dashv \vdash A \vee B \mapsto C
$$


Frankel, L. (1986). Mutual causation, simultaneity and event description. Philosophical Studies, 49, 361-372.

Graßhoff, G., \& May, M. (2001). Causal regularities. In W. Spohn, M. Ledwig, \& M. Esfeld (Eds.), Current issues in causation (pp. 85-114). Paderborn: Mentis.

Halpin, J. F. (2003). Scientific law: A perspectival account. Erkenntnis, 58, 137-168.

Hausman, D. (1998). Causal asymmetries. Cambridge: Cambridge University Press.

Hintikka, J. (1973). Distributive normal forms in first-order logic. In Logic, language games and information (pp. 242-286). Oxford: Clarendon Press.

Hume, D. (1999/1748). An enquiry concerning human understanding. Oxford: Oxford University Press.

Kim, J. (1973). Causation, nomic subsumption, and the concept of event. Journal of Philosophy, $70,217-236$.

Lemmon, E. J. (1978/1965). Beginning logic. Indianapolis: Hackett.

Mackie, J. L. (1974). The cement of the universe. A study of causation. Oxford: Clarendon Press.

Mackie, J. L. (1993/1965). Causes and conditions. In E. Sosa \& M. Tooley (Eds.), Causation (pp. 33-55). Oxford: Oxford University Press.

May, M. (1999). Kausales Schliessen. Eine Untersuchung über kausale Erklärungen und Theorienbildung. Ph.D. thesis, Universität Hamburg, Hamburg.

Mellor, D. H. (1995). The facts of causation. London: Routledge.

Molnar, G. (1969). Kneale's argument revisited. The Philosophical Review, 78, 79-89.

Price, H. (1992). Agency and causal asymmetry. Mind, 101, 501-520.

Ragin, C. C. (2000). Fuzzy-set social science. Chicago: University of Chicago Press.

Reichenbach, H. (1956). The direction of time. Berkeley: University of California Press.

Russell, B. (1986). The philosophy of logical atomism. In J. G. Slater (Ed.), The philosophy of logical atomism and other essays. The collected papers of Bertrand Russell (pp. 157-244). London: George Allen \& Unwin.

Russell, B. (1992/1913). On the notion of cause. In J. G. Slater (Ed.), The collected papers of Bertrand Russell. Logical and philosophical papers 1909-13 (Vol. 6, pp. 190-210). London: Routledge.

Sanford, D. (1976). The direction of causation and the direction of conditionship. The Journal of Philosophy, 73, 193-207.

Suppes, P. (1970). A probabilistic theory of causality. Amsterdam: North Holland.

Swartz, N. (2003). The concept of physical law. (2nd ed.). http://www.sfu.ca/philosophy/ physical-law/.

van Fraassen, B. (1989). The charybdis of realism. Epistemological implications of Bell's inequality. In J. T. Cushing \& E. McMullin (Eds.), Philosophical consequences of quantum theory (pp. 97-113). Notre Dame: University of Notre Dame Press.

Woodward, J. (2003). Making things happen. Oxford: Oxford University Press.

$\mathrm{Xu}$, M. (1997). Causation in branching time (I): Transitions, events and causes. Synthese, 112, 137-192.

Zangwill, N. (2003). Negative properties, determination and conditionals. Topoi, 22, 127-134. 